\title{
Characterization of (0-3) piezocomposite materials for transducer applications
}

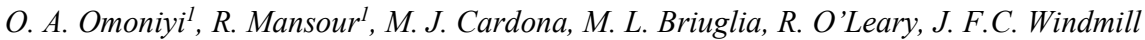 \\ Centre for Ultrasonic Engineering, Electronic \& Electrical Engineering Dept., \\ Bioacoustics Group, University of Strathclyde \\ Glasgow, Scotland, United Kingdom \\ Oluwaseun.omoniyi@strath.ac.uk
}

\begin{abstract}
In this study, we have developed and characterized two different (0-3) piezoelectric composite materials with potential to be used in sensing applications. The composite materials were made using Polydimethylsiloxane (PDMS) as the polymer matrix with Barium Titanate $\left(\mathrm{BaTiO}_{3}\right)$, and Lead Zirconate Titanate (PZT51) as the dielectric fillers. Thin film samples of the (0-3) piezocomposites were prepared using a solution mixing and spin coating method to produce composites with (0-3) connectivity pattern and layer thickness of $100 \mu \mathrm{m}$. The microstructure of the piezocomposites were analyzed using a scanning electron microscope to determine the connectivity structure and homogeneity of the piezocomposites. The mechanical properties of the composites were determined using the method of Oliver and Pharr. FTIR analysis was used to determine the effects of the fillers on the structure of the piezocomposite. The average piezoelectric $d_{33}$ coefficient of the piezocomposites were also measured using the laser vibrometer technique and determined to be $30 \mathrm{pm} / \mathrm{V}$ for the piezocomposite consisting of Barium Titanate $\left(\mathrm{BaTiO}_{3}\right)$ and $32 \mathrm{pm} / \mathrm{V}$ for the piezocomposite consisting of Lead Zirconate Titanate (PZT51).
\end{abstract}

Keywords- Piezoelectric; Ceramic-Polymer; Spin coating; UV curable; Composite; FTIR; XRD; SEM

\section{INTRODUCTION}

Recently, there has been a growing need to develop advanced functional materials to produce highly functioning transducers. To meet such needs, the functional materials should be able to perform different functions such as sensing and actuation simultaneously within a given system. Materials which exhibit piezoelectric properties have been used in transducer technology by harnessing the sensing and actuation function of such materials. Much research has gone into developing a class of highly functional materials based on the piezoelectric effect. These piezocomposite materials comprise of a piezoelectric ceramic mixed with a polymer to produce a combination which offers much promise in the manufacturing of sensing and actuating devices due to the combined properties of each constituent material such as the piezoelectric properties of the ceramic and the mechanical flexibility of the polymer. Some of these can be integrated with metals or polymer composites for structural health monitoring (SHM), and in producing electromechanical transducers such as pressure sensors, hydrophones, and vibration sensors [1,2]. A piezocomposite consists of two phases and can be classified according to the way both

The work was supported by the Engineering and Physical Sciences Research Council (EPSRC) under grant EP/L022125/1, and by the European Research Council under the European Union's Seventh Framework Programme (FP/2007-2013) / ERC Grant Agreement n. [615030]. phases are connected dimensionally e.g. (1-, 2-, or 3-) into 10 structures; $0-0,0-1,0-2,0-3,1-1,1-2,1-3,2-2,2-3$, and 3-3 [3]. Although each structure provides different advantages, one structure which offers much promise is the (0-3).

In a composite with ( $0-3)$ connectivity, a ceramic filler is mixed with a three-dimensionally connected polymer phase. These composites can be used to easily fabricate large flexible thin sheets, produce applications in large scale and shaped to conform to variety of shapes [2]. Composites with $(0-3)$ structure were first discovered by Kitayama Pauer and Sugawara [4-5], this was achieved using PZT as the filler material and polyurethane as the matrix. These early (0-3) composites were reported to have low $d_{33}$ values [6]. Different materials such as $\mathrm{PBTiO}_{3}$ ceramic and eccogel polymers, PT-BF powder, have been used to fabricate these piezocomposites [7]. Several types of flexible piezoelectric composites consisting of $\mathrm{PbTiO}_{3}$ powder and chloroprene rubber, $\mathrm{BaTiO}_{3}$ and Norlands Polymer have been developed [8-10], obtaining $d_{33}$ in the range of 1.2 to $3.8 \mathrm{pm} / \mathrm{V}$.

In this paper, we focus on the development and characterization of a (0-3) piezocomposite with Barium Titanate (BT) and PZT as the filler materials and PDMS as the polymer matrix.

\section{MATERIALS AND METHODS}

The materials used in this experiment include commercially available Polydimethylsiloxane (PDMS), Sylgard 184, Silicon elastomer curing agent (SigmaAldrich), silver paint, $\mathrm{BaTIO}_{3} 500 \mathrm{~nm}$ nanoparticles (US research nanomaterials INC, USA) and Lead Zirconate

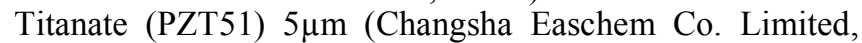
China).

\section{A. Material Synthesis and Screen Printing}

The fabrication process is carried out in two steps. The first step involved synthesizing the Polymers. Silicon elastomer curing agent was mixed with Silicon elastomer at a ratio 1:9. Silicon elastomer curing agent is needed to start the polymerization process when exposed to high temperature. The mixture was put in a THINKY AER-250 mechanical mixer and mixed for $3 \mathrm{~min}$ at $1500 \mathrm{rpm}$ and de-foamed for $2 \mathrm{~min}$ at $1200 \mathrm{rpm}$. The process removed all air bubbles within the mixture and ensured proper mix between the filler and matrix. A PDMS mixture with viscosity of $3500 \mathrm{cP}$ was obtained.

The second step involved making the (0-3) piezocomposites with the polymer synthesized above. $60 \%$ 
w/w BT was added to $40 \% \mathrm{w} / \mathrm{w}$ PDMS and $60 \% \mathrm{w} / \mathrm{w}$ PZT 51 was added to $40 \% \mathrm{w} / \mathrm{w}$ PDMS. Both composites were prepared separately and mixed in a THINKY AER-250 mechanical mixer for $3 \mathrm{~min}$ at $1500 \mathrm{rpm}$ and de-foamed for 2 min at $1200 \mathrm{rpm}$. This process was repeated twice and sonicated for 30 mins. Thin film samples of each piezocomposite were obtained using a spin coating technique. Samples were spin coated using an Ossila spin coater at $2000 \mathrm{rpm}$ for $10 \mathrm{~s}$ to give a layer thickness of $100 \mu \mathrm{m}$. Although efforts were made to obtain a uniform thickness layer all through, this was unachievable due to the filler particle sizes used and the goal to have thin film samples of approximately $80-100 \mu \mathrm{m}$. The piezocomposite samples were cured under high temperature at $150{ }^{\circ} \mathrm{C}$ for $2 \mathrm{~h}$ using an oven (Heraeus thermo scientific).

Finally, thin film samples of sizes $3 \mathrm{~cm}$ by $2 \mathrm{~cm}$ were cut out and silver paint coated on both sides of the piezocomposites. All samples were polarized in silicon oil heated to a constant temperature of $100{ }^{\circ} \mathrm{C}$ under a voltage of up to $9 \mathrm{kV}$ for $2 \mathrm{~h}$.

\section{EXPERIMENTAL RESULTS AND DISCUSSIONS}

\section{A. Morphology}

The microstructure of each piezocomposite sample was investigated using a table-top scanning electron microscope at an accelerating voltage of $15 \mathrm{kV}$ (SEM, Hitachi TM1000, Krefeld, Germany). In Fig. 1(a), the cross-section area of the composite with PZT and PDMS is shown. Thick clusters of the filler is observed with non-uniform dispersion. This result shows a poor degree of bonding and poor adhesion between the PZT filler and PDMS which also indicates that the filler may be inter-connected giving rise to a non (0-3) structure. This could be as a result of the high PZT wt. \% used, the particle size $(\sim 5 \mu \mathrm{m})$ and the solution mixing method used in preparing the composite. In Fig 1(b). The micrograph confirms the (0-3) structure of the composite and shows a higher degree of dispersion than the PZT composite. Here, the BT filler is seen to be densely and uniformly and more homogenous with no apparent agglomeration. Although, some spaces were observed (arrows) in the composite, this is possibly due to the mechanical mixing technique used. (a)

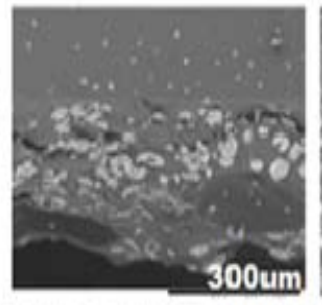

(b)

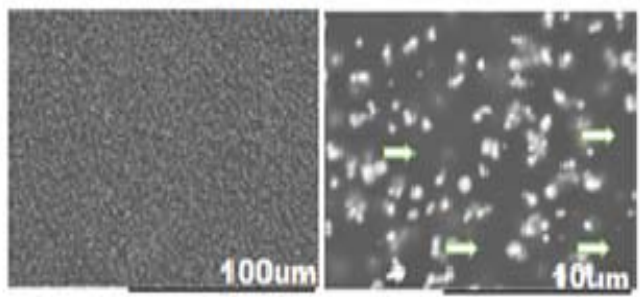

Fig. 1(a). Shows the scanning electron micrographs of the surface area of the composite material containing $60 \mathrm{wt} . \%$ PZT and $40 \mathrm{wt} . \%$ PDMS. (b). Shows the scanning electron micrographs of the surface area of the composite material containing 60 wt. $\%$ BT nanoparticles and 40 wt. $\%$ PDMS.

\section{B. FTIR Analysis}

FTIR spectra of the composite material were obtained using a Tensor II Bench ATR-IR (Bruker). Each spectrum was background subtracted. The samples were scanned in an inert atmosphere over a wave number range of 4000-400 $\mathrm{cm}^{-1}$ with a resolution better than $0.01 \mathrm{~cm}^{-1}$.
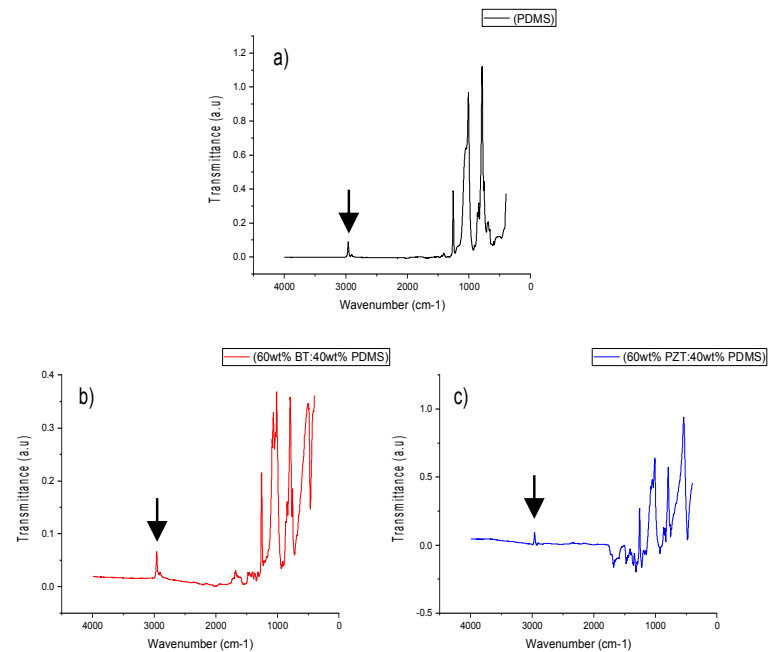

Fig. 2. Shows the FTIR spectra of piezocomposite with PDMS. a) shows the FTIR of pure PDMS; $b$ ) indicates the FTIR spectra of PDMS combined with $60 \%$ wt of BT and c) reveals the spectra for PDMS combined with $60 \%$ PZT nanoparticles.

Fig. 2(a) shows only PDMS and exhibits IR peaks at 789$796 \mathrm{~cm}^{-1}\left(-\mathrm{CH}_{3}\right.$ rocking and Si-C stretching in $\left.\mathrm{Si}-\mathrm{CH}_{3}\right)$, $1020-1074 \mathrm{~cm}^{-1}$ (Si-O-Si stretching), $1260-1259 \mathrm{~cm}^{-1}\left(\mathrm{CH}_{3}\right.$ deformation in $\mathrm{Si}-\mathrm{CH}_{3}$ ), and 2950-2960 $\mathrm{cm}^{-1}$ (asymmetric $\mathrm{CH}_{3}$ stretching in $\mathrm{Si}-\mathrm{CH}_{3}$ ). When the BT and PZT nanoparticles are combined with PDMS (Fig. 2b and 2c), the characteristic peaks of PDMS are maintained and the fingerprint region shows extra signals, which represent the bonds between the piezocomposite and the polymer. The combination between nanoparticles and polymer does not alter the structure of PDMS.

\section{XRD Analysis}

XRD analysis was performed using a Bruker D2 Phaser diffractometer at ambient temperature with an operating voltage of $30 \mathrm{kV}$ and an operating current of $10 \mathrm{~mA}$. The detection was ensured by $10.5 \mathrm{~mm} \mathrm{Ni}$ low Beta filter, $2.5^{\circ}$ Soller Slits and LynxEye 1D Detector.

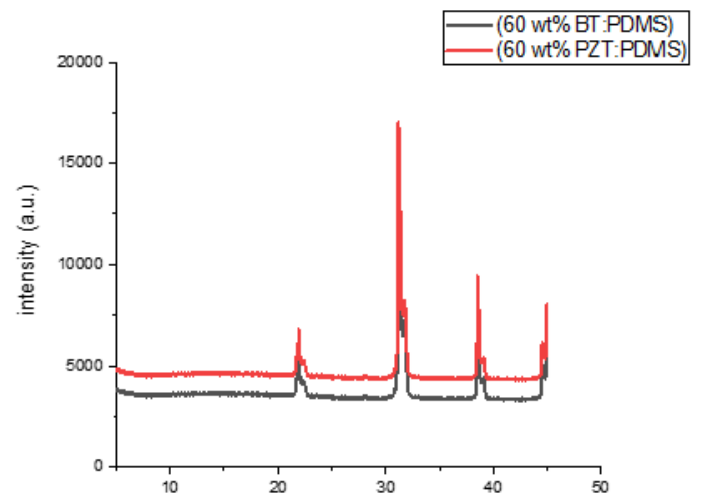

Fig. 3. Shows the XRD pattern for two piezocomposite samples. The red signal represents a sample with $60 \%$ wt. of PZT while the black spectra indicates a sample formed of $60 \%$ of BT. 


\section{Piezoelectric properties}

The piezoelectric properties of the (0-3) piezocomposites were investigated using a microscanning laser Doppler vibrometer (Polytec MSA100-3D, Waldbronn, Germany) technique [13]. The composites were glued firmly on a metal block to prevent bending of the substrate. A sinusoidal AC voltage of $10 \mathrm{~V}$ was applied across the axis of charging and the resulting displacement along the poling axis was measured.

The $d_{33}$ coefficient of the (0-3) piezocomposites consisting of BT and PZT filler particles in PDMS was investigated using the direct displacement mode of the laser vibrometer. The result obtained under single point scans is shown in Fig. 4. The mechanical displacement of each piezocomposite is observed with BT: PDMS having a thickness resonance frequency at $15 \mathrm{kHz}$ while the piezocomposite with PZT: PDMS exhibited maximum displacements at $6 \mathrm{kHz}$, and 11 $\mathrm{kHz}$ respectively. A full surface scan with a grid of 1000 scan points was performed below each resonant frequency obtained during the single point scan to obtain a more accurate result. The piezocomposite BT: PDMS gave an average $d_{33}$ of $30 \mathrm{pm} / \mathrm{V}$, while the piezocomposite with PZT: PDMS exhibited an average $d_{33}$ of $32 \mathrm{pm} / \mathrm{V}$.

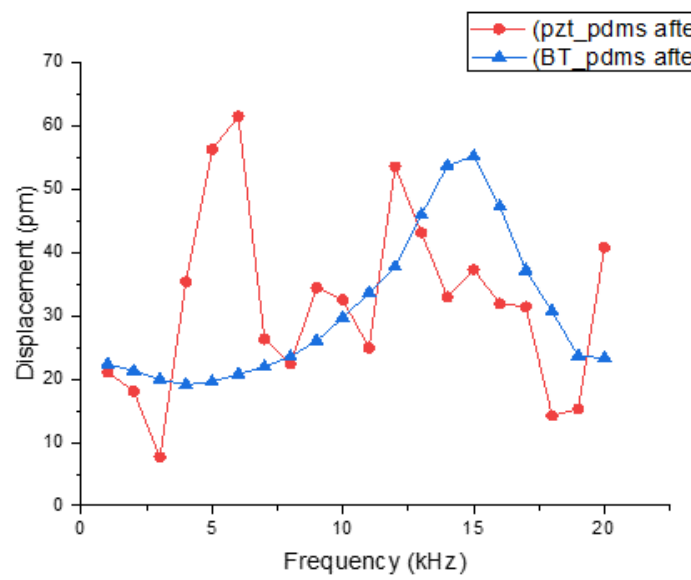

Fig. 4. Shows the magnitude of mechanical displacement as a function of a driving sinusoidal voltage of $10 \mathrm{Vp}$-p delivered over a frequency range between 1 and $20 \mathrm{kHz}$.

\section{E. Mechanical Properties}

The mechanical properties of the piezocomposites consisting of BT and PZT as fillers were investigated using the method of Oliver and Pharr. Indentation modulus of the samples was evaluated using an MFT-3D Nanoindenter. A maximum load of $460 \mu \mathrm{N}$ was applied to the samples and a $6 \times 6$ array of indentations separated by $15 \mu \mathrm{m}$ was carried out to a fixed depth of $1 \mu \mathrm{m}$ in order to reduce influence from neighboring indentations and to avoid the substrates influence on the mechanical properties. Indentation modulus $(\mathrm{n}=36)$ was measured and shown in table 1 .

Table 1. Average indentation modulus E obtained.

\begin{tabular}{|l|c|}
\hline Piezocomposites & Average Indentation modulus (MPa) \\
\hline PDMS: BT & $19.95 \pm 2.20$ \\
\hline PDMS: PZT & $26.08 \pm 9.50$ \\
\hline
\end{tabular}

\section{CONCLUSIONS}

Different piezocomposite materials comprising of piezoceramic fillers in polymer matrix is presented in this work. The piezocomposites are designed with a (0-3) structure and fabricated using $\mathrm{BaTiO}_{3}$, PZT nanoparticles, and PDMS as polymer matrix. The piezocomposites were characterized using different techniques to determine the structural, piezoelectric and mechanical properties of the material. The structural analysis of the composite revealed that the polymer matrix with BT fillers closely exhibits the (0-3) structure while that consisting of PZT revealed a composite with more than one connectivity pattern, a combination of a (0-3) structure and non (0-3) structure. This could be as a result of the size of the PZT nanoparticle, the high $60 \% \mathrm{w} / \mathrm{w}$ ratio and the solution mixing technique used. Both XRD and FTIR analysis showed that both BT and PZT fillers did not alter the structure of PDMS.

Furthermore, the 3D LDV experiments showed the piezoelectric nature of both piezocomposite samples. It was observed that at higher frequencies $(6 \mathrm{kHz}, 1 \mathrm{kHz}$, and 15 $\mathrm{kHz}$ ) all the samples vibrate with higher amplitudes which could be due to the samples having a uniform thickness and homogeneity across different regions or different regions having agglomeration of nanoparticles as observed in the SEM micrographs. The mechanical properties of the composite material were tested using the nanoindentation method, the indentation modulus obtained showed the material exhibits plastic behavior which could be due to high BT and PZT loading. The thin and flexible structure of this piezocomposites coupled with the piezoelectric properties shows that this material offers promise as a functional material which can be used in sensing applications. Although, further work is required to investigate the dielectric properties of these piezocomposites and to further enhance their $d_{33}$ value.

\section{ACKNOWLEDGMENT}

The authors would like to thank the staff at the University of Strathclyde Centre for Ultrasonic Engineering group.

\section{REFERENCES}

[1] W. H. Duan, Q. Wang and S. T. Quek, "Applications of Piezoelectric Materials in Structural Health Monitoring and Repair: Selected Research Examples. Materials 2010, 3, 5169-5194; doi:10.3390/ma3125169

[2] R. Y. Ting, Composite Piezoelectric Materials for Transduction, Appl. Acoust., vol 41, pp. 325-335, 1994

[3] Babu, I. "Piezoelectric composites : design, fabrication and performance analysis Eindhoven": Technische Universiteit Eindhoven (2013). DOI: 10.6100/IR760468

[4] T. Kitayama and Sugawara, Rept. Prof. Gr. Inst. Elec. Comm. Eng. Japan, pp. CPM 72-77, 1972. (in Japanese)

[5] L. A. Pauer, "Flexible piezoelectric material," presented at IEEE Int. Conv. Rec., 1973.

[6] W. B. Harrison, "Flexible piezoelectric organic composites," in Proc. Workshop on Sonar Transducer Mat., Naval Research Laboratory, Arlington, VA, 1976.

[7] H. Banno and S. Saito, "Piezoelectric and dielectric properties of composites of synthetic rubber and PbTiO3 or PZT,'Jpn. J. Appl. Phys., vol. 22, pp.67-69,1983.

[8] H. Banno, "Recent developments of piezoelectric ceramic products and composites of synthetic rubber and piezoelectric ceramic particles," Ferroelectrics, vol. 50, pp.3-12,1983. 
[9] H. Banno, K. Ogura, H. Sobue, and K. Ohya, "Piezoelectric and acoustic properties of piezoelectric flexible composites," Jpn. J. Appl. Phys., vol. 26, pp.153-155,1987.

[10] R. Mansour, O. A. Omoniyi, R. O'Leary and J. F. C. Windmill, "Fabrication of Ultraviolet-Curable Piezoelectric Composite for Sensor and Actuator Applications," 2018 IEEE SENSORS, New Delhi, 2018, pp. 1-4. https://doi.org/10.1109/ICSENS.2018.8589943

[11] J. R. Giniewicz, K. Duscha, R. E. Newnham, and A. Safari, "0- 3 Composites for hydrophone applications," presented at IEEE Int. Symp. Appl. Ferroelect., Bethlehem, PA, 1986.

[12] Domingo-Roca, R., Jackson, J. C., \& Windmill, J. F. C. Bioinspired 3D-printed piezoelectric device for acoustic frequency separation. In Proceedings of IEEE Sensors (Vol. 2017-December, pp. 1-3).
Institute of Electrical and Electronics Engineers Inc. https://doi.org/10.1109/ICSENS.2017.8234384

[13] Yao, K., \& Tay, F. E. H. Measurement of longitudinal piezoelectric coefficient of thin films by a laser-scanning vibrometer. IEEE Transactions on Ultrasonics, Ferroelectrics, and Frequency Control. https://doi.org/10.1109/TUFFC.2003.1182115 